Recherches en didactique des langues et des cultures

Les cahiers de l'Acedle

2| 2006

Recherches en didactique des langues. Actes du colloque de Lyon

\title{
Littérature et didactique
}

Marie-Françoise Narcy-Combes

\section{(2) OpenEdition \\ 1 Journals}

Édition électronique

URL : http://journals.openedition.org/rdlc/5445

DOI : $10.4000 /$ rdlc.5445

ISSN : 1958-5772

Éditeur

ACEDLE

Référence électronique

Marie-Françoise Narcy-Combes, "Littérature et didactique », Recherches en didactique des langues et des cultures [En ligne], 2 | 2006, mis en ligne le 15 mars 2019, consulté le 11 septembre 2019. URL: http://journals.openedition.org/rdlc/5445; DOI : 10.4000/rdlc.5445

Ce document a été généré automatiquement le 11 septembre 2019

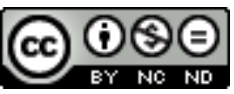

Recherches en didactique des lanques et des cultures is licensed under a Creative Commons AttributionNonCommercial-NoDerivatives 4.0 International License 


\title{
Littérature et didactique
}

\author{
Marie-Françoise Narcy-Combes
}

\section{Préparation aux concours de recrutement et responsabilité épistémologique}

1 Ce texte se propose d'examiner ce qui se passe lorsqu'un enseignant-chercheur en didactique des langues se trouve confronté aux contradictions qui se font jour lorsqu'on lui confie la préparation à l'épreuve dite de didactique au concours de l'agrégation interne d'anglais. Entre l'état du savoir aujourd'hui en didactique, les exigences propres à l'épreuve qui nécessite entre autres un savoir encyclopédique en littérature et civilisation, et les préoccupations des candidats, il était difficile de se repérer sans mener une réflexion sur la manière de prendre en compte ces différents paramètres de façon à respecter les intérêts de chacun sans pour autant se renier soi-même. Il s'agissait d'offrir aux stagiaires une formation qui leur permette d'aborder l'épreuve avec sérénité tout en gardant le recul que donne la responsabilité épistémologique.

\subsection{La démarche}

Le référentiel de cette épreuve ${ }^{1}$ montre que cinq capacités vont être évaluées, qui, toutes, concernent à première vue le didacticien. En effet, être capable d'analyser un corpus de documents supports, de sélectionner ceux qui vont permettre d'atteindre les objectifs d'apprentissage, de construire un parcours et d'élaborer les tâches pertinentes afférentes, enfin évaluer la validité du dispositif mis en place et le développement de l'interlangue des apprenants font bien partie des missions que se fixe la didactique des langues. La cinquième capacité, communiquer, plus technique, concerne davantage le praticien pédagogue, et moins la réflexion didactique.

Un examen plus attentif du référentiel, à la lumière des théories didactiques montre, dans un second temps, qu'il s'agit plutôt d'un montage institutionnel destiné à vérifier la présence d'un certain nombre de compétences chez les candidats que d'une réflexion didactique telle que pourraient la concevoir les chercheurs du domaine. En effet, sur le 
terrain, le praticien didacticien commence par déterminer les objectifs d'apprentissage pour les apprenants dont il a la responsabilité, c'est-à-dire pour un public dont il connaît les besoins dans un contexte donné et en fonction de contraintes spécifiques. Le tableau ci-dessous permet de visualiser les contradictions liées aux contraintes de l'épreuve, notamment la nécessité de proposer un choix de documents en dehors de tout contexte d'enseignement, ce qui, en didactique, est déjà contraire à la pratique. "De l'analyse des documents découle donc la définition des objectifs. C'est ce qui garantit une véritable intégration des objectifs culturels et linguistiques" indique le rapport sur les épreuves orales de 2004. Or construire une séquence d'enseignement/apprentissage dans le paradigme institutionnel actuel qui se veut constructiviste et communicationnel, c'est d'abord fixer des objectifs, ensuite réfléchir aux moyens de les atteindre.

Tableau 1 - Le référentiel de l'épreuve orale de didactique à l'agrégation interne d'anglais et la démarche didactique : tableau comparatif

\begin{tabular}{|l|l|}
\hline $\begin{array}{l}\text { Référentiel de l'épreuve orale de l'agrégation } \\
\text { interne d'anglais }\end{array}$ & Démarche didactique \\
\hline Analyser les documents & $\begin{array}{l}\text { Analyser le contexte d'enseignement / } \\
\text { apprentissage }\end{array}$ \\
\hline Choisir le niveau, les objectifs, les documents & Déterminer les objectifs \\
\hline $\begin{array}{l}\text { Construire le parcours d'enseignement } \\
\text { apprentissage }\end{array}$ & $\begin{array}{l}\text { Construire le parcours d'enseignement } \\
\text { apprentissage }\end{array}$ \\
\hline Mettre en place les évaluations & Choisir les supports adaptés \\
\hline Communiquer & Mettre en place les évaluations \\
\hline & Communiquer, faciliter, guider \\
\hline
\end{tabular}

\subsection{Les contenus}

4 Le premier point, dans la définition de l'épreuve, consiste à analyser les documents mis à disposition, leur spécificité, leur potentiel pour l'enseignement de la langue, et pour l'enseignement d'une culture également, au sens large du terme, comprenant non seulement les conduites et valeurs propres à un ensemble d'individus, mais aussi et force est de le constater - surtout, les artefacts liés à cette culture. Comme on le voit, mettre cette dimension à la première place revient à considérer que les contenus sont prioritaires. Le point de départ de cette démarche, comme dans les approches antérieures à l'avènement du communicatif, c'est l'objet de culture: la langue, la littérature essentiellement, la civilisation accessoirement. La centration sur les contenus est révélatrice de la confusion entre l'acquisition d'un savoir savant et celle de L2 et ne tient pas compte de la nécessaire transposition didactique. Le didacticien aujourd'hui commencera par l'analyse du contexte d'enseignement / apprentissage. Quelles sont les caractéristiques du public? Quels sont ses besoins? Quelles sont les contraintes institutionnelles dont il est nécessaire de tenir compte? De quelles ressources dispose-t- 
on? Le travail de mise en place d'un dispositif sera gouverné par le principe de réalité, et suivant les théories de Vygotski sur la zone proximale de développement, il conviendra d'aller chercher l'apprenant où il se trouve pour le conduire un peu plus loin, dans la direction qu'il souhaite suivre. Dans ce cas-là, il n'est pas possible de commencer par les supports d'enseignement, dont le rôle est de fournir les éléments linguistiques et extralinguistiques nécessaires à l'apprentissage.

En second lieu, les candidats sont invités à choisir le niveau, les objectifs, et à sélectionner les documents supports pour la séquence qu'ils vont prévoir, avec la difficulté bien sûr de devoir sélectionner une classe virtuelle. Le principe de réalité est occulté dans cette approche, car tout enseignant expert sait que ce qui fonctionne dans un contexte donné ne fonctionnera pas nécessairement dans un environnement différent. Les objectifs ne peuvent être fixés que par rapport à un public donné, à ses besoins et ses motivations. À partir de là, et seulement à ce moment-là, il devient possible de construire la séquence d'enseignement / apprentissage autour de tâches qui permettront aux apprenants de construire leur apprentissage. Les supports, selon leur nature, leur genre, la langue qu'ils proposent, seront alors choisis par l'enseignant de façon à étayer la progression et à faciliter l'apprentissage, en relation étroite avec les tâches proposées.

Dans le bas du tableau, la définition de l'épreuve rejoint les positions de la didactique en intégrant l'évaluation dans le parcours d'apprentissage, ce qui implique de savoir ce que chacun met sous les mots, car le dernier critère, le rôle de l'enseignant comme communicateur, semble bien refléter une vision uniquement transmissive de l'enseignement / apprentissage.

7 Par ailleurs, la lecture de ce rapport stipule que l'épreuve ne se borne pas à évaluer les compétences des candidats en didactique des langues, mais exige également des connaissances encyclopédiques en "littérature, civilisation, histoire, géographie, politique, etc., $d u$ monde anglophone". Le rapport souligne en outre que les candidats font preuve "d'une grande confusion d'esprit et confondent fantastique, merveilleux, étrange, féerique, etc." Il apparaît donc clairement que les candidats doivent être des spécialistes non seulement de didactique, mais également, et peut-être surtout, de littérature et de civilisation.

\subsection{Les objectifs}

Partant de ce constat, le formateur se trouve devant un dilemme fréquent lorsque la formation engage la réussite à un concours de recrutement ou à un examen : comment assurer que les étudiants reçoivent un enseignement de qualité, tout en mobilisant les moyens nécessaires pour rendre possible la réussite aux épreuves ? La théorie de l'effet boomerang (washback effect) nous montre que le poids de l'examen peut souvent pervertir la qualité de la formation proposée. Les étudiants réclament davantage des outils et des recettes pour réussir l'épreuve, plus qu'ils ne souhaitent développer des compétences réelles et assurer leur développement professionnel et personnel, démontrant de ce fait un investissement réduit. Si cette demande reçoit un accueil favorable, la formation perd beaucoup de sa pertinence et de son sens. S'il n'en est pas du tout tenu compte, les étudiants ne sont pas satisfaits, et la formation ne sera pas davantage efficace puisqu'elle ne répondra pas à leurs attentes. Nous touchons là à un problème de responsabilité épistémologique, tant du point de vue des candidats aux concours, que des formateurs (voir à ce propos Narcy-Combes J.-P., 2005). 
9 Pour répondre à ce problème, la démarche d'analyse en didactique aide le praticien. L'auteur de ces lignes a jusque-là mené sa réflexion dans le contexte du secteur Lansad. Or on associe souvent la langue académique aux autres formes de langue de spécialité, et, en poussant plus loin l'analogie, il est possible de considérer la littérature ou la civilisation comme des spécialités à part entière avec leurs genres, leurs types de discours, leur terminologie spécifiques. Ceci nous renvoie à l'une des questions fondamentales de l'enseignement des langues aux spécialistes d'autres disciplines : quel rôle donner au spécialiste de la discipline ? La réponse est, lorsque cela est possible, de combiner les compétences de l'un à celles du spécialiste de langue, en s'efforçant d'associer langue et contenu. Nous avons émis l'hypothèse que ce modèle théorique ( Content and Language Learning, cf. Benoit, 2004) pouvait être transposé dans le contexte de l'enseignement de la didactique des langues, ce qui nous a conduit à proposer une formation à deux voix. Partant du postulat que le didacticien ne peut qu'être aidé par une bonne analyse de la langue et des supports, nous avons décidé de faire intervenir un spécialiste de littérature et un spécialiste de didactique. Le premier avait pour tâche de sensibiliser les étudiants à la spécificité des documents supports possibles d'une part (iconographiques, textuels et vidéo) et aux différents genres littéraires d'autre part (poésie, théâtre, texte narratif). Le second avait celle de mettre en évidence la transposition didactique possible autour de tâches (Task Based Language Learning).

La particularité d'un enseignement de la littérature tient au fait que, pour les élèves, il ne s'agit pas uniquement d'acquérir des compétences linguistiques, mais aussi de s'approprier un savoir de spécialité: on se situe bien au niveau de CALP (Cognitive Academic Language Proficiency) la langue académique, reconnue comme langue de spécialité par les chercheurs aujourd'hui. Cela nous indique que ce type d'enseignement ne peut s'adresser qu'à des apprenants qui ont développé des capacités cognitives qui leur permettent de traiter l'information à ce niveau de compétence (Robinson, 2002). Le positionnement théorique que prendra le didacticien alliera l'approche par tâches et l'acquisition de la langue par les contenus.

\section{Mise en place d'une séquence d'enseignement / apprentissage en littérature : le cadre théorique de l'apprentissage par les tâches}

11 Nous l'avons dit plus haut, pour construire une séquence, il convient que l'enseignant se fixe des objectifs cohérents avec le paradigme constructiviste et communicationnel qui est celui de l'institution aujourd'hui, puis qu'il réfléchisse aux moyens de les atteindre. Pour ce faire, il lui faudra mette en place des tâches, ce qui suppose une théorisation implicite sur l'acquisition langagière. On se situe au niveau des processus. Il lui faudra, d'autre part, mobiliser des ressources, ce qui suppose une théorisation implicite sur la langue. On est au niveau du produit. Nous postulons qu'il existe une relation transductive entre le langage, le contenu et la pensée, c'est-à-dire une relation dans laquelle un élément ne peut exister sans les deux autres (Narcy-Combes J.-P., 2005). Il se dégage en recherche aujourd'hui un consensus sur le fait que l'acquisition de la L2 est liée à la culture et au savoir, et donc suppose un contenu véhiculé par des paroles que l'on objective en langue. Cela n'est pas sans implications pour l'enseignement de la langue à travers la littérature. 
12 Il y a apprentissage lorsque les élèves s'approprient des contenus et produisent de la langue compréhensible dans une production sollicitée par l'enseignant (Ellis, 1994). Les premières tâches proposées seront proches des contenus proposés, pour s'en libérer progressivement. Ellis (2003), que nous suivrons ici, parle de macro-tâches (les simulations et jeu de rôles intégrant les quatre compétences) et de micro-tâches (un travail a posteriori sur des points de détail qui posent problème). Les premières sont communicatives, les secondes sont individuelles. Nous postulons qu'il est difficile de séparer le sens (langue outil), de la forme (langue système) et éviterons de proposer des tâches décontextualisées, même si nous considèrerons que dans les macro-tâches la focalisation est sur le sens, alors qu'elle est sur la forme dans le cas des micro-tâches.

Ellis encore insiste sur la nécessité de proposer des tâches authentiques de degré de complexité varié, c'est-à-dire qui correspondent à des activités qui pourraient avoir lieu dans la réalité, ce qui n'est pas vraiment le cas des activités souvent proposées en classe, comme de répondre à des questions sur un texte ou de résumer ce texte, de remplir une grille de compréhension, etc. Ces tâches mettent en jeu des processus dont il convient que le praticien prenne la mesure : comprendre, manipuler, produire, interagir en utilisant la langue. Elles ont une double dimension: cognitive (évaluer l'information, réfléchir, déduire, établir des liens, classer, organiser) et linguistique. Le résultat (la production de l'apprenant) est le reflet de cette double dimension. Si l'enseignant donne pour consigne d'écrire un poème "à la manière de", le résultat de la tâche sera la combinaison d'un savoir linguistique adéquat et de la capacité à comprendre et maîtriser le genre poétique. Ceci montre que le résultat diffère quelque peu des objectifs de l'enseignement des langues que l'on peut exprimer ainsi : c'est le développement des capacités de réception et de production langagières. Ainsi, le résultat de la tâche peut être atteint sans que l'objectif le soit. Or ce qui intéresse l'enseignant de langue, c'est l'objectif, c'est-à-dire qu'il y ait acquisition langagière, et ce qui intéresse les élèves la plupart du temps, c'est le résultat, c'est-à-dire la réalisation concrète d'une production pas nécessairement linguistique, mais ce sont les processus cognitifs et linguistiques à l'œuvre pour atteindre le résultat qui sont cruciaux. En d'autres termes, le résultat de la tâche importe peu pour l'acquisition linguistique, mais il semble que lorsque les élèves utilisent la langue pour mener la tâche à bien, il y ait acquisition.

14 La progressivité s'effectue du simple au complexe, de l'explicite à l'implicite, du concret à l'abstrait. Il convient que le praticien tienne compte non seulement, comme nous l'avons vu plus haut, de la spécificité des documents, de leur nature, de la complexité du code qu'ils utilisent, mais également de leur complexité cognitive, de leur dépendance du contexte (les références culturelles par exemple) et de la familiarité des élèves avec ce type de document. Pour la réalisation de la tâche il conviendra d'en avoir déterminé au préalable les supports, la complexité, le mode et le domaine de discours et les solutions possibles qui peuvent être ouvertes, c'est-à-dire qu'on peut admettre plusieurs réponses différentes, ou fermées, c'est-à-dire qu'une seule réponse est possible.

La mise en œuvre se fera en trois étapes, avant, pendant et après la tâche.

16 La première étape, avant la tâche, consiste à expliquer l'activité prévue, sa nature, les conditions de sa réalisation. (De combien de temps les élèves disposent-ils pour la mener à bien ? Comment vont-ils s'y prendre ?) Il est possible, mais non obligatoire, à cette étape de donner un exemple, ou un modèle. 
deuxième étape, pendant la tâche, est de faire respecter les conditions de réalisation, proposer un accompagnement sur la langue, sur les contenus.

La troisième étape, après la tâche, est le moment où retour, prolongements, correction et évaluation sont envisagés.

\section{Spécificités de trois genres en littérature : la poésie, le théâtre, la narration}

\subsection{La poésie}

19 La poésie fait partie de l'univers des humains et permet une ouverture vers une autre appréhension du monde, un autre éclairage sur le quotidien. Même si l'on se place dans une approche communicative, les êtres humains ont d'autres choses à communiquer, d'autres messages à faire passer que de l'utilitaire. La poésie peut permettre aux élèves de ressentir et d'exprimer toute une gamme d'émotions et de sentiments de manière inhabituelle tout en maniant la langue avec précision. En effet, la forme est première en poésie, ce qui en fait un art à part. Le poète s'exerce à la pratique contrôlée de la langue. Dans les poèmes, parce que peu de mots sont utilisés pour le message communiqué, chaque mot est important. Parce que chaque mot est important, plusieurs techniques sont utilisées pour augmenter leur pouvoir évocateur : sons, rimes, images, contrastes, juxtapositions. La langue est une surprise, ce qui dynamise la force de mémorisation. La répétition, de mots, de vers, de groupes de vers joue également sur la mémoire : mais à la différence de ce qui se passait lors de la répétition de drills structuraux, le mot, ou le groupe de mots répétés prennent chaque fois une résonance nouvelle, associant ainsi la forme au sens. Enfin la structure même du poème, son organisation spatiale, l'utilisation des sons, de l'orthographe et de la syntaxe conduisent les élèves à travailler la langue tout en s'essayant à communiquer un message qui les implique profondément.

\subsection{Le théâtre}

Un des éléments qui caractérise le théâtre c'est la pluralité de lectures, donc d'interprétations que permet le passage de l'écrit à la scène, comme en témoignent les traductions diverses des œuvres d'auteurs du passé, de Sénèque à Brecht en passant par Shakespeare et Molière. La spécificité du genre comprend l'organisation du texte sur la page, avec les indications scéniques et les types d'échanges entre personnages : silence, monologues, stichomythies, et les divisions en actes, scènes et tableaux. Cette organisation sous-tend l'organisation spatio-temporelle de l'œuvre théâtrale, en tension entre le temps du récit, celui de la représentation et le temps métaphorique ou mythique dont tout texte théâtral est porteur. La forme nous offre une entrée vers le sens. Pour l'explorer plus avant, le praticien amènera ses élèves à s'intéresser à la fable (la succession chronologique des évènements imaginés ou racontés par l'auteur) et à l'intrigue (la succession des mécanismes entre l'exposition, le nœud, les péripéties et le dénouement) qu'il faudra retrouver, et parallèlement au personnage et à son rôle dans la pièce, comme distinct de l'acteur qui l'interprète. Ce dernier produit un discours, est luimême présent dans le discours des autres personnages de la pièce, ce qui nous conduit à l'étude des énoncés et de l'énonciation. Qui parle, à qui, à l'intérieur et à l'extérieur du 
texte théâtral ? Au théâtre, parole et action sont indissociables : dire, c'est aussi faire, et toute parole est potentiellement un déclencheur d'actions.

S'exprimer par le théâtre est une activité kinesthésique, le corps tout entier participe à l'expression d'un message. Par-là les élèves peuvent être amenés à prendre conscience que la communication ne se limite pas à des mots mis bout à bout, que l'expression verbale d'un message allie la justesse de l'intonation et l'adéquation de l'accentuation, que le sens passe aussi par le geste, et les signes que donnent le contexte ou la situation. Le jeu dramatique peut leur permettre de s'exprimer plus longuement qu'ils ne le font généralement dans un enseignement plus traditionnel, d'exprimer des émotions et des sentiments sans passer par la description ou la narration, tâches plus scolaires.

\subsection{Le texte narratif}

Lorsqu'on décide de mettre les élèves face à un texte narratif, se rapprocher de la réalité c'est prendre en compte le plaisir de la lecture. La rencontre avec le texte ne se réduit pas au repérage d'informations, et il est même possible de dire que ce n'est pas l'objectif du lecteur qui prend un récit et se plonge dans la lecture. Les sources de plaisir sont liées à la mise en œuvre de l'imaginaire, et au travail interprétatif auquel se livre le lecteur lorsqu'il construit le sens du texte entre mémorisation et projection. Cette négociation permanente du sens fait écho à ce qui se passe en apprentissage des langues, et permet donc de développer des compétences transdisciplinaires. Accroître le plaisir de la lecture, c'est développer les capacités d'inférences. Les connaissances contextuelles sur le récit sont autant de clins d'œil qui rassurent et réjouissent le lecteur. C'est aussi accroître la capacité à procéder à des mises en réseau, ce qui n'est possible qu'en développant des savoirs et des compétences sur le lexique, mais aussi sur les genres et les types de discours. Comprendre les ruptures par rapport à des règles convenues, à un scénario attendu, implique d'avoir intégré ces règles ou ces scénarios. Le lecteur sera ainsi conduit à apprécier les utilisations du temps et de l'espace, de la structure du texte, des différents niveaux de narration et des effets produits. Puis il prolonge sa lecture en l'inscrivant dans un cadre plus vaste, et construit ainsi son propre contexte culturel de référence.

\section{Transposition didactique}

La didactisation (certains disent pédagogisation) donnera lieu à des approches diverses en fonction du public, de ses capacités linguistiques, de ses intérêts et de ses motivations. Ceci ne signifie pas qu'il soit impossible ou peu souhaitable d'initier à la littérature un auditoire peu motivé par le sujet, simplement l'enseignant expert s'y prendra de façon différente de celle dont il use lorsqu'il a affaire à une classe a priori intéressée par le sujet. Ainsi, la nouvelle de Nadine Gordimer dont nous traitons plus loin pourra être l'aboutissement d'un travail thématique sur l'Afrique du Sud avec certaines catégories d'élèves, ou le support à la découverte des techniques narratives avec d'autres.

Il semble que deux processus essentiels entrent en jeu dans l'acquisition d'une L2 (Narcy-Combes J.-P., 2005) : le repérage (noticing) et la gestion du sens (deep processing). Ils occupent une place centrale et sont en lien avec l'ensemble des opérations concernant le passage de l'input à l'output. De plus, comme le repérage est nativisé, c'est-à-dire que les observations faites par l'apprenant seront analysées largement en fonction de sa culture et de sa langue d'origine, la médiation de l'enseignant sera nécessaire au moment de la 
présentation des contenus afin de le sensibiliser à ce qu'il n'aura pas été à même de percevoir.

La démarche combinera donc chaque fois ces deux activités essentielles de gestion du sens et de repérage formel. Par exemple, il est admis aujourd'hui que l'apprentissage par cœur est une aide à l'entraînement mémoriel sur le plan phono-articulatoire, mais aussi pour les blocs lexicalisés et la syntaxe. Pour donner du sens à un apprentissage par cœur, il sera nécessaire de le relier à des activités qui le justifient, comme de jouer une pièce de théâtre, réciter un poème ou chanter. Dans une perspective constructiviste, les élèves seront guidés sur un parcours d'observation et de recherche d'informations, d'analyse de ces données, d'émission d'hypothèses, pour aboutir à une production. Ce qui importe ici c'est que l'apprenant observe et mémorise à sa façon ce qu'il a observé, qu'il s'agisse de règles au cours d'un travail analytique ou de blocs lexicalisés. Dans le cas d'un texte littéraire, il est important de prendre conscience qu'en raison de la charge implicite de ce type de texte, le repérage purement factuel est inadapté, car l'intérêt que représente la rencontre avec la littérature est alors perdu. À chacune de ces étapes des manques, des besoins, des conflits pourront émerger qui susciteront un recours à l'expertise pour les résoudre. Ces problèmes ne sont pas prévisibles, ce qui requiert de l'enseignant une grande souplesse. Son rôle est d'attirer l'attention sur ce qui n'aura pas été observé au cours de l'apprentissage par la découverte. Ainsi, les exercices, dans un dispositif constructiviste, sont vus comme des tâches a posteriori pour répondre à un besoin révélé par une activité initiale (Narcy-Combes J.-P., 2005).

Dans le second degré, les enseignants proposent pour l'essentiel des tâches à objectifs déterminés et ciblés, ce qui peut se justifier lorsque le public concerné est bien identifié et relativement homogène, avec la réserve qu'il n'est alors que peu impliqué dans les décisions le concernant, ce qui peut poser problème pour la motivation. On y rencontre en outre deux écueils à chaque extrême d'un continuum : ou bien le travail sur la langue est vu a priori, dans ce cas la pratique langagière est réduite, et par voie de conséquence les acquisitions le sont également ( il n'y a pas de gestion mentale compensatoire), ou à l'autre extrême, l'enseignant pratique une approche communicative pure et propose des tâches "communicatives authentiques" en pensant que cela suffira à assurer les acquisitions, ce qui n'est pas le cas, comme l'ont montré les travaux de recherche antérieurs.

\subsection{La poésie}

Pour sensibiliser les élèves au discours poétique il est possible d'imaginer de leur faire réécrire, par groupes de trois ou quatre, le poème proposé dans la langue courante. La macro-tâche permet, d'une part, une négociation du sens à travers les interactions, d'autre part, une prise de conscience de la nature des différents types de discours par la comparaison qui s'impose de façon quasi naturelle entre ce que l'on perd et ce que l'on gagne dans l'un et l'autre cas.

Cette tâche peut jouer le rôle de déclencheur pour le développement d'activités centrées sur le genre poétique et il est alors possible de proposer une série de tâches ciblées consistant à repérer, classer, comparer, expliquer, pour aboutir à de mini productions.

- Sur le sens :

- faire repérer l'énonciateur, le destinataire du poème, les éléments de caractérisation ;

- faire repérer les actions, les lieux, le temps. 
Sur le lexique :

- faire exprimer sous diverses formes la préférence, le compliment, le conseil, etc. pour explorer les divers champs sémantiques;

- sensibiliser les élèves à la valeur métaphorique des mots, repérage des images et des symboles débouchant sur un travail interprétatif ;

- inviter les élèves à créer des associations sens / mots pour les amener à repérer et apprécier les associations poétiques et comprendre les effets de surprise suscités par la créativité des auteurs.

- Sur la musicalité, le rythme, les échos :

- faire rechercher les rimes, les allitérations, les assonances, les parallélismes, les répétitions ;

- les classer par catégories grammaticales et analyser les effets produits pour aboutir à de mini productions personnelles et aussi à une mise en bouche, au plaisir de dire le poème.

\section{- Sur la langue :}

- sensibiliser les élèves à la différence de nature de la langue : le poème, par l'économie de mots grammaticaux qu'il présente, esquive la fonctionnalité de la langue. Demander aux élèves de la rétablir suscite un travail de réflexion sur la langue. Mais il est aussi possible de proposer d'utiliser préfixes, suffixes et morphèmes pour fabriquer d'autres mots, de travailler sur les contrastes, les contraires, les connotations positives ou négatives qui peuvent varier selon les cultures.

Lire, aimer un poème implique la participation active du lecteur, et, de sa part, un travail d'interprétation personnelle dont le résultat varie en fonction du contexte socioculturel et de l'histoire de chacun. On permettra à chaque élève d'exprimer cette dimension à travers une tâche invitant à l'interprétation, par exemple un essai en langue étrangère sur le thème : "J'aime ce poème parce que..."

Enfin, l'aboutissement de l'apprentissage peut être envisagé sous la forme d'un travail de création poétique "à la manière de".

\subsection{Le théâtre}

À partir de la pluralité du texte, il est possible de proposer aux élèves une macro-tâche interactive. Le jeu consiste pour un groupe à interpréter une scène devant le reste de la classe qui prend le rôle du metteur en scène et intervient en ce sens. Se mettent alors en place diverses interprétations, au cours d'un travail de compréhension du texte, pour aller vers une représentation théâtrale qui satisfasse l'ensemble du groupe. L'activité implique pour les élèves d'utiliser la capacité à argumenter, discuter, défendre un point de vue. Les "metteurs en scène" sont conduits à prendre en compte les indications scéniques ou didascalies d'une manière naturelle. Un va-et-vient constant entre le texte et son organisation, d'une part, et le jeu scénique, d'autre part, est indispensable pour la réussite de la tâche, conduisant à un repérage des spécificités formelles du genre. $\mathrm{Au}$ moment des interactions, l'enseignant est en retrait. Il prend des notes, et pourra proposer les micro-tâches nécessaires à un travail de précision que l'analyse de ces notes mettra en évidence, et qu'il n'est pas vraiment possible de prévoir à l'avance. Cependant, il est probable que la demande viendra des acteurs eux-mêmes. Les expériences menées dans le primaire montrent que pris par le jeu, ce sont les élèves qui viennent demander au professeur son aide pour résoudre les problèmes auxquels ils se trouvent confrontés au cours de l'élaboration de la tâche. On a vu ainsi le passage à l'écrit se faire 
naturellement, en réponse aux besoins exprimés. Pour mémoriser leur texte, les élèves ont eu besoin d'une trace écrite, qu'ils ont d'abord créée eux-mêmes : une retranscription phonétique à partir de ce qu'ils entendaient. Puis l'une d'entre eux a voulu savoir: "Maîtresse, comment ça s'écrit en vrai ?", déclenchant ainsi la curiosité de tout le groupe, et des réflexions métacognitives bien plus susceptibles de contribuer à la construction de savoir que des impositions externes.

Comme pour la poésie, le travail de mémorisation et le travail phonologique nécessaires au théâtre, sont favorables pour l'acquisition de la L2, d'autant plus qu'ils ont un sens dans ce contexte.

Après ce type de tâche, voir la pièce jouée par des acteurs professionnels c'est accéder à une autre interprétation possible qui pourra servir de base de discussion sur les choix du metteur en scène par rapport aux attentes, aux compréhensions, et aux découvertes des élèves. Ils auront une vision de l'intérieur du texte dramatique, ce qui leur permettra de s'exprimer en connaissance de cause. L'entreprise peut paraître ambitieuse, et l'est certainement. Mais là encore l'expérience montre que c'est un haut niveau d'exigence, allié à une approche humaniste prenant en compte les réactions des élèves, qui permet la réussite de l'enseignement / apprentissage (Dubet \& Durubella, 2001).

\subsection{Le texte narratif}

Nous l'avons dit plus haut, la découverte de la littérature ne peut être dissociée de la notion de plaisir. Pour apprécier un récit, il convient de sensibiliser les élèves à l'importance :

- de la notion de contexte ;

- de la connaissance des genres et des scénarios littéraires ;

- de la compréhension de la structure de l'œuvre ;

- de la compréhension de la langue ;

et des liens entre cette compréhension avec le plaisir de la découverte, le plaisir d'apprendre, la jubilation que donne le sentiment de connivence avec l'auteur.

À partir de la nouvelle de Nadine Gordimer, "Is There Nowhere Else Where We Can Meet ?", nous allons proposer une démarche dont nous ne garderons que les éléments transférables.

\subsubsection{Le contexte}

On demande aux élèves une recherche documentaire en amont sur :

- l'Afrique du Sud au temps de l'Apartheid;

- l'auteur Nadine Gordimer ;

et par équipes de quatre de présenter en classe lors de courts exposés de 10mns les résultats de leur recherche.

41 En leur fournissant bibliographie, articles et adresses Internet, ils seront conduits à synthétiser l'information, et à la présenter à l'oral. Ils rencontreront également ainsi deux types de discours : informatif et littéraire.

\subsubsection{Rencontre avec le texte}

- Inférence à partir du titre 

susciter des interprétations fort diverses, et seul le contexte l'éclaire.

- Lecture du texte jusqu'à la ligne 80

On fera repérer sous forme de tableau les personnages ( $\mathrm{He}$, She, le nom, les éléments de caractérisation), le narrateur, l'auteur, le lieu, le temps.

La tâche consistera à écrire un article pour le journal local relatant l'événement. Que faut-il ajouter au texte littéraire pour le rendre acceptable ? Quelle langue utiliser pour le rédiger ? La tâche permet de mesurer l'écart entre le texte informatif et le texte littéraire dans une démarche comparative.

\subsubsection{Structure du texte}

Il s'agira de rédiger la suite de la nouvelle en tenant compte :

- des éléments constitutifs de l'histoire ;

- de la construction du récit ;

- de l'interprétation (projection du scénario).

L'imagination et la créativité des élèves seront sollicitées. Des micro-tâches de repérage pourront être proposées en fonction des besoins sur l'organisation des paragraphes, leur nombre, leur contenu, les phrases et mots-clés, sur les temps utilisés et sur l'écriture : les mots qui réfèrent aux couleurs, aux sensations, les métaphores et comparaisons jouent un rôle clé dans ce texte. Après les avoir fait repérer aux élèves, on peut poursuivre le travail avec d'autres couleurs, d'autres sensations, d'autres métaphores pour aboutir à la rédaction d'un petit texte descriptif "à la manière de ..." dans une perspective formative.

47 On peut imaginer une création collective, ou par groupe. Dans ce dernier cas, il est important que d'une manière ou d'une autre l'ensemble des élèves ait accès aux productions des autres groupes.

\subsubsection{Rencontre avec la littérature}

À ce stade, on donne aux élèves la fin de l'histoire. En quoi se démarque-t-elle de ce que les élèves ont imaginé ? C'est en cela que la littérature est création : elle rompt avec les attentes du lecteur. Un travail métacognitif peut maintenant être effectué sur la création littéraire.

Les élèves connaissent maintenant bien la nouvelle de Nadine Gordimer. Ils ont été sensibilisés à la spécificité du genre, aux caractéristiques de la langue employée, aux effets du contexte sur l'interprétation. Ils ont enrichi leurs connaissances linguistiques et sollicité leur créativité et leur imagination. Ils sont maintenant capables de réaliser la tâche suivante : réécrire la scène de la rencontre, moment phare de la nouvelle, vu du point de vue de l'indigène, à la première personne du singulier.

On évaluera leur capacité à respecter le genre de la nouvelle, la cohérence de l'ensemble, la correction, la richesse et la précision de la langue, l'originalité et la créativité. 


\section{Conclusion}

\subsection{Mise en évidence des compétences nécessaires en plusieurs domaines pour mener à bien le projet}

51

Ce que nous avons tenté de présenter ici ne sont que les conclusions auxquelles nous sommes parvenus à cette étape de notre réflexion. Nous sommes conscients que chaque point abordé pourrait être développé sur un chapitre, voire un ouvrage complet. En effet, la complexité des contenus et des compétences en jeu le justifierait. Nous avons examiné trois genres littéraires : la poésie, le théâtre et le texte narratif. Chacun de ces genres exige des compétences d'expert sur ses spécificités. Ce n'est que s'il est bien informé de ces contenus que le didacticien peut proposer une démarche d'enseignement / apprentissage qui tiendra compte à la fois

- des élèves, de leurs aptitudes et de leurs motivations: les tâches proposées devront être réalistes et susciter l'implication;

- des objectifs dont le but ultime demeure l'acquisition d'une langue étrangère en milieu institutionnel ;

- des contraintes cognitives, affectives et institutionnelles qui pèsent sur cette acquisition.

Il lui faudra pour cela une expertise en ce qui concerne la nature et le rôle des tâches, et la capacité de les intégrer de façon efficace dans la construction d'une séquence d'enseignement / apprentissage.

\subsection{Peut-on vraiment entraîner les enseignants à cette démarche ? Quels sont les obstacles? Comment y parvenir?}

Pour le didacticien l'évaluation finale fait partie intégrante de la formation et permet d'engager avec les participants un dialogue propice à une co-construction des savoirs. Nous avons donc proposé aux agrégatifs de s'exprimer en fin de parcours (cf. document en annexe), offre qu'ils ont décliné sans explications, ce qui a laissé les deux enseignants-chercheurs perplexes quant à l'implication et au niveau de réflexion didactique des agrégatifs, et réduits aux hypothèses non validables faute de rétroaction.

Si ce que se propose de valider l'épreuve de l'agrégation interne est véritablement les compétences didactiques des enseignants de langue, et non un savoir encyclopédique sur la littérature et la civilisation du pays dont la langue est enseignée, il serait souhaitable qu'elle corresponde davantage à ce que préconisent les connaissances du domaine. Nous proposerions la constitution d'un dossier thématique mettant en œuvre une démarche didactique et pédagogique incluant une mise en œuvre sur le terrain et une analyse des effets de cette mise en œuvre. Ceci n'alourdirait pas pour autant la charge du jury. Il serait parfaitement envisageable de sélectionner une liste d'une dizaine de thèmes chaque année. L'épreuve orale, qui pourrait être maintenue dans les temps de l'épreuve actuelle, serait une soutenance de ce dossier. Ceci pourrait conduire les candidats à s'impliquer davantage dans la préparation de cette épreuve, et à intégrer véritablement les concepts didactiques qui pour le moment et pour bon nombre d'entre eux semblent rester lettre morte. Les connaissances en littérature ou civilisation n'en souffriront pas pour autant car il deviendra possible d'exiger des futurs agrégés une maîtrise accrue du sujet traité. Cette démarche d'évaluation terminale nous paraît donner aux formateurs 
les moyens nécessaires pour qu'eux-mêmes et les étudiants puissent échapper au piège $\mathrm{du}$ clonage et des recettes toutes faites. Les apprenants de didactique viendraient en formation avec de vraies questions suscitées par des problèmes rencontrés dans la réalité de la salle de classe, et donc avec une volonté de comprendre et une motivation un peu plus soutenue.

Ce travail nous a permis enfin de souligner les décalages entre les théories et la pratique qui interpellent le chercheur en didactique des langues. Prendre un document pour point de départ d'une construction de séance fait courir le risque de le présenter à des élèves qui ne sont pas prêts à l'accueillir. Décider de faire de la littérature avec certains élèves peut conduire l'enseignant à se limiter au communicatif et à l'évènementiel, ce qui ne présente pas d'intérêt par rapport au support. C'est alors que recul et responsabilité épistémologiques s'imposent.

\section{BIBLIOGRAPHIE}

Benoit, W. (2004). L'approche par tâches dans l'apprentissage de l'anglais de spécialité :

opérationnalisation contrôlée dans l'enseignement supérieur. Thèse de doctorat : université de Nantes.

Dubet, F. \& Durubella, M. (2001). L'hypocrisie scolaire. Paris : Éditions du Seuil.

Ellis, R. (1994). The Study of Second Language Acquisition. Oxford: OUP.

Ellis, R. (2003). Task-based Language Learning and Teaching. Oxford: OUP.

Gordimer, N. (1952). "Is There Nowhere Else Where We Can Meet? In The soft voice of the serpent. New York: Simon \& Schuster.

Narcy-Combes, J.-P. (2005). Didactique des langues et TIC : vers une recherche-action responsable. Paris: Ophrys.

Robinson, P. (2002). Individual Differences and Instructed Language Learning. Amsterdam /

Philadelphia : John Benjamins.

Vygotski, L. S. (1997). Pensée et langage. Paris : La dispute / SNEDIT.

\section{ANNEXES}

\section{Enquête auprès des agrégatifs}

L'étude des rapports de jury et l'expérience des préparations des années précédentes ont conduit à l'hypothèse suivante.

La littérature est une spécialité, la didactique en est une autre. Une préparation optimale pour l'épreuve de didactique au concours de l'agrégation telle qu'elle est définie aujourd'hui devrait combiner les compétences de spécialistes de l'une et l'autre disciplines. 
Cette hypothèse a conduit à proposer cette année une formation à deux voix, en littérature et didactique. Nous aimerions à ce stade savoir comment vous avez accueilli cet ajustement.

Pouvez-vous ci-dessous nous faire part de vos commentaires concernant :

- la part dévolue à chacune des spécialités ;

- les apports réciproques littérature / didactique ;

- l'efficacité globale de la préparation.

\section{NOTES}

1. Rapport du jury du concours 2001, pp. 141-143.

\section{RÉSUMÉS}

Cet article s'appuie sur une réflexion sur une pratique d'enseignement. Il s'est peu à peu imposé que la littérature étant une spécialité, la démarche adoptée dans le secteur des langues pour spécialistes d'autres disciplines devait être pertinente dans ce domaine également. Une des conclusions auxquelles j'étais parvenue était la nécessité de travailler en tandem avec les spécialistes en contenu. Au cours de l'année universitaire 2004-2005, nous avons pu mettre en place une intervention à deux voix dans le cadre de la formation des professeurs d'anglais dans le second degré, le spécialiste de littérature mettant en évidence les spécificités des genres et des types de discours de sa spécialité, le spécialiste en didactique proposant une démarche et un dispositif susceptible de sensibiliser les élèves de lycée et de leur permettre d'accéder à cette discipline.

Ce sont les implications de cette formation que cet article se propose d'étudier ici, en prenant en compte les avancées récentes dans les deux domaines de spécialité.

This article is about how language teaching methodology can help when it comes to associating language learning and content. If literature is seen as content, it can be dealt with in the same way as any other content, such as specialised content in the field of English for Specific Purposes where the specialist for the content works hand in hand with the language teacher. From this perspective we postulate that the most reliable and efficient way to deal with teaching how to teach literature is by combining the skills of a specialist in literature and those of a specialist in language teaching. The former is to deal with the specific genres and discourses of the speciality; the latter is to offer various techniques most likely to appeal to learners at a secondary school level, which enable them to understand what literature is. We will examine the implications such a stand may have on teaching and learning English while taking into account recent research in both fields. 
INDEX

Mots-clés : didactique des langues, littérature, formation, dispositif

Keywords : teaching methodology, literature, education, system

\section{AUTEUR}

\section{MARIE-FRANÇOISE NARCY-COMBES}

Marie-Françoise Narcy-Combes est maître de conférences à l'université de Nantes. Ses enseignements concernent les LEA (Langues Etrangères Appliquées) et la didactique des langues et sont en relation avec son domaine de recherche : didactique du secteur Lansad(LANgues pour le Spécialiste d'Autres Disciplines), didactique de l'interculturel.

Courriel : mf.narcycombes[at]wanadoo.fr 